\title{
AMDORAP: Non-targeted metabolic profiling based on high-resolution LC-MS
}

Hiroki Takahashi ${ }^{1}$, Takuya Morimoto ${ }^{1,2}$, Naotake Ogasawara ${ }^{1}$ and Shigehiko Kanaya ${ }^{\text {* }}$

\begin{abstract}
Background: Liquid chromatography-mass spectrometry (LC-MS) utilizing the high-resolution power of an orbitrap is an important analytical technique for both metabolomics and proteomics. Most important feature of the orbitrap is excellent mass accuracy. Thus, it is necessary to convert raw data to accurate and reliable $\mathrm{m} / \mathrm{z}$ values for metabolic fingerprinting by high-resolution LC-MS.

Results: In the present study, we developed a novel, easy-to-use and straightforward $\mathrm{m} / \mathrm{z}$ detection method, AMDORAP. For assessing the performance, we used real biological samples, Bacillus subtilis strains 168 and MGB874, in the positive mode by LC-orbitrap. For 14 identified compounds by measuring the authentic compounds, we compared obtained $\mathrm{m} / \mathrm{z}$ values with other LC-MS processing tools. The errors by AMDORAP were distributed within \pm 3 ppm and showed the best performance in $m / z$ value accuracy.

Conclusions: Our method can detect $\mathrm{m} / \mathrm{z}$ values of biological samples much more accurately than other LC-MS analysis tools. AMDORAP allows us to address the relationships between biological effects and cellular metabolites based on accurate $\mathrm{m} / \mathrm{z}$ values. Obtaining the accurate $\mathrm{m} / \mathrm{z}$ values from raw data should be indispensable as a starting point for comparative LC-orbitrap analysis. AMDORAP is freely available under an open-source license at http://amdorap.sourceforge.net/.
\end{abstract}

\section{Background}

Metabolomics is defined as technology designed to give us the broadest, least biased insight into the richly diverse population of small molecules present in living things [1]. Understanding cells at the levels of the transcriptome and metabolome provides insight into the network of complex biological regulations [2-5]. Metabolites within cells have the diverse range of chemical and physical properties and the wide range of those concentrations [6]. To achieve metabolomics, two analytical platforms, i.e., mass spectrometry (MS) and nuclear magnetic resonance spectroscopy (NMR), have been widely used $[7,8]$. Chromatography-MS technologies play a central role in measuring the complex biological samples. Out of these, liquid chromatography-MS (LC-MS) is capable of detecting a broader range of metabolites than other MS technologies such as gas chromatography-MS and capillary electrophoresis-MS [9]. Therefore, LC-MS has become

\footnotetext{
* Correspondence: skanaya@gtc.naist.jp

${ }^{1}$ Graduate School of Information Science, Nara Institute of Science and

Technology, 8916-5 Takayama, Ikoma, Nara 630-0192, Japan

Full list of author information is available at the end of the article
}

more widely used in metabolomics analysis. An orbitrap mass analyzer is the most recent addition to the set of tools that can be applied to identification, characterization and quantitation of components in biological systems since its commercial introduction in 2005 [10]. Orbitrap-based MSs have been proven to be a powerful tool in proteomics because they have $\approx 100000$ resolving power at a mass-to-charge ratio $(\mathrm{m} / z) 400[11,12]$. The most important feature of the orbitrap is that it can stably maintain excellent mass accuracy without re-calibration, and does not require the use of calibration standards [13]. Accurate $m / z$ values can be used to define molecular formulae in putative identification of metabolites $[7,14]$. Consequently, in the field of non-targeted metabolomics, those instruments make it possible to identify candidate molecular formulae from mass differences in measured $m / z$ values $[15,16]$.

Public databases of chemical compounds such as ChEBI [17], HMDB [18], KEGG [19], KNApSAcK [20] and PubChem [21] provide suitable compounds for each molecular formula without measuring reference samples in advance. The species-metabolite relationship database 
KNApSAcK, for example, can easily narrow down candidates from accurate masses according to the species information or the type of ion adducts [22,23]. Several molecular ion adducts should be considered especially when the ionization of molecules in samples is performed by electrospray ionization $[24,25]$. Once given, the accurate $m / z$ values can lead to the information of molecular formulae and candidate compounds by considering the mass differences, the appropriate ion adduct and the species together. However, it should be noted that structural isomers and stereoisomers with the same mass require the complicated chromatographic separation before mass analyzing [7].

Allen et al. [26] analyzed several "silent mutants" of yeasts (viable mutants with no obvious phenotype) by comparing extracellular metabolites using LC-MS data collected in a non-targeted approach. In preprocessing the LC-MS data, they skipped peak detection and annotation schemes typically used for such data; instead, they reduced data into a single aggregate MS vector and applied clustering and machine learning methods. Their study demonstrated the effectiveness of metabolic fingerprinting of extracellular extracts by non-complicated preprocessed data. Metabolic fingerprinting with the exclusion of $\mathrm{m} / \mathrm{z}$ resolution, however, is impossible to get more insight from same data sets. The high-resolution of the orbitrap can be exploited in metabolic fingerprinting. In NMR or Fourier transform ion cyclotron resonance based MS (FT-ICR-MS), valid information about metabolic regulation in biological samples can be obtained by resolving power alone, even without any chromatographic separation [27].

An easy-to-use, flexible and automated tool is a key to success in metabolomics studies. This is particularly the case in high-resolution MS analyses mainly because of the data size. Our aim is to estimate more accurate $m / z$ values and extract interesting $\mathrm{m} / \mathrm{z}$ values from raw data in comparative LC-orbitrap analysis. In the present study, we describe a novel straightforward $\mathrm{m} / z$ detection method, "AMDORAP" (Accurate $\mathbf{m} / \mathbf{z}$ detection method for LCorbitrap) for high-resolution MS (e.g., the orbitrap) by taking advantage of its stable mass accuracy.

\section{Implementation}

Several freely available frameworks for analyzing LC-MS data sets have been developed [28]. The typical MS data processing workflow comprises multiple stages, including filtering, feature detection, alignment and normalization. In MZmine 2 [29,30], peak alignment across samples, for example, follows peak detection for individual samples. The Bioconductor package XCMS [31,32] mainly consists of peak detection, peak matching and retention time alignment. A common concept shared by widely used methods, including MZmine 2 and XCMS, is that peak detection step for both $\mathrm{m} / \mathrm{z}$ and retention time dimensions is executed for an individual sample, or scan, followed by an alignment (or merging) step across samples. The most important reason for using highresolution MS is to obtain more accurate $m / z$ values from biological samples. That makes it possible to identify correct candidate molecular formulae from mass differences alone. Since the orbitrap can determine $\mathrm{m} / \mathrm{z}$ values extremely accurately, we assumed that $\mathrm{m} / z$ values derived from compounds with the same compositional formula, including structural isomers and stereoisomers, should be robust with respect to retention time and differences between samples.

In this study, we developed the preprocessing method, AMDORAP (Accurate $\mathbf{m} / \mathbf{z}$ detection method for LCorbitrap) written in the $\mathrm{R}$ programming language [33] in order to attain the quick comparison of metabolic profiling by high-resolution MS. Figure 1 illustrates the AMDORAP procedure, which comprises three steps:

1. Collect data points with intensities larger than a threshold for all samples.

2. Group collected data points by $m / z$ closeness, and estimate representative $m / z$ values for individual $m / z$ groups.

3. Extract ion chromatograms for the $m / z$ list.

The main idea motivating this procedure is that peak picking and alignment steps of $m / z$ values should be performed in a single step. In the following section, the AMDORAP performance was assessed using data sets in the positive mode from two Bacillus subtilis strains 168 and MGB874 [34].

\section{Results and Discussion}

\section{Sample preparation and experimental conditions}

In order to assess the AMDORAP performance, we performed the experiments and then prepared the biological data sets. Two Bacillus subtilis strains, wild-type 168 and the genome reduced strain MGB874 [34], were used for metabolome analysis. The cells were cultured at $37^{\circ} \mathrm{C}$ to an $\mathrm{OD}_{600}$ value of 4.0 in the early stationary phase of growth, in Spizizen's minimal medium (SMM) [35] supplemented with $0.5 \%$ glucose, $5 \mu \mathrm{g} / \mathrm{ml}$ tryptophan, $20 \mu \mathrm{g} /$ $\mathrm{ml}$ methionine and trace elements [36]. Metabolite extraction was performed according to Takahashi et al. [23]. The culture media were passed through a $0.4 \mu \mathrm{m}$ HTTP filter (Millipore). Residual cells on the filter were washed twice with HPLC grade water and then immersed in $2 \mathrm{ml}$ of methanol. After incubation at $4^{\circ} \mathrm{C}$ overnight, the extracts were centrifuged at $9000 \times g$ at $4^{\circ} \mathrm{C}$ for $10 \mathrm{~min}$, filtered through $0.2 \mu \mathrm{m}$ PTFE membrane (Advantec), evaporated 


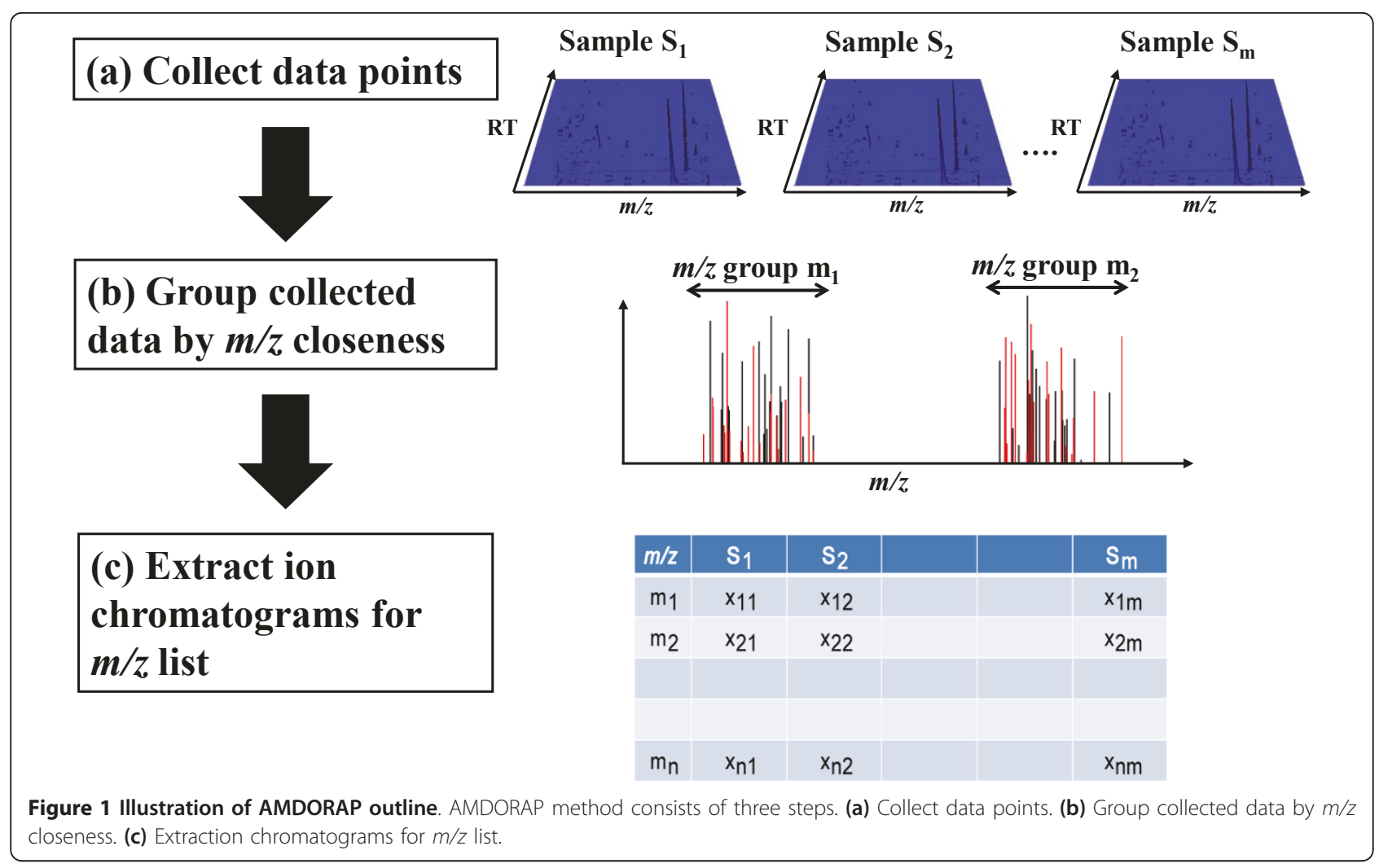

at room temperature and stored at $-80^{\circ} \mathrm{C}$. The extracts were dissolved in $200 \mu \mathrm{l}$ of $80 \%$ methanol before analysis in the LC-orbitrap.

Mass analysis was performed using a Paradigm MS4 system (Michrome BioResources) coupled to an LTQorbitrap XL-HTC-PAL system (Thermo Fisher Scientific). All experimental events were controlled by Xcalibur software version 2.0.7 (Thermo Fisher Scientific). HPLC was performed under the conditions as described by Iijima et al. [37]. Samples were injected into to a TSKgel column ODS-100V $(4.6 \times 250 \mathrm{~mm}, 5 \mu \mathrm{m}$; TOSOH $)$. Water (HPLC grade; solvent A) and acetonitrile (HPLC grade; solvent $\mathrm{B}$ ) were used as the mobile phase with $0.1 \% \mathrm{v} / \mathrm{v}$ formic acid. The gradient program was as follows: $3 \%$ B to $97 \%$ B (45 min), $97 \%$ B (5 min) and 10\% $B(10 \mathrm{~min})$. The flow rate was set to $0.5 \mathrm{ml} / \mathrm{min}$. The ESI setting was as follows: spray voltage $4.5 \mathrm{kV}$ and capillary temperature $350^{\circ} \mathrm{C}$ for the positive ionization mode. Nitrogen sheath gas and auxiliary gas were set at 60 and 20 arbitrary units, respectively. A full MS scan was performed in the $m / z$ range $70-1500$ at a resolution of 60000 . Simultaneously, top three $\mathrm{MS}^{2}$ spectra within each full MS scan were gained by the linear ion trap at a collision energy of $35 \mathrm{eV}$. Thermo Fisher mass spectrometry RAW files were converted from profile mode into centroid mode using the ReAdW program [38].

\section{AMDORAP performance \\ Collection of data points}

Figure $2 \mathrm{~b}$ shows the intensity distribution of a centroid data from B. subtilis strain 168 . The total number of data points was 1694959 (1945 scans within 45 minutes). The top $1 \%$ of the data (represented by a red dot in Figure 2a) could explain $99.7 \%$ of the total variance of all data points. Thus, almost all data obtained by the LCorbitrap can be considered as background noise. Here, we assumed that the top $1 \%$ of the data was detected ions, and the other $99 \%$ was noise for each sample in the collecting step. Figure 3 shows the total ion chromatograms and two dimensional map. The total ion chromatogram of top $1 \%$ of data highly correlates with that of all data (in Figure 3a) and then top 1\% of data is extensively scattered in both dimensions (in Figure 3b), suggesting that top $1 \%$ of data can explain the characterization of all data with respect to intensities and dimensions.

Grouping collected data points and estimation of representative $\mathrm{m} / \mathrm{z}$ values for individual groups

As the second step, all collected $m / z$ values are grouped by closeness, i.e., if differences between the neighboring $\mathrm{m} / \mathrm{z}$ values are within $5 \mathrm{ppm}$ (default setting), they are grouped together. There is no limit of data points within one $m / z$ group as long as this constrain is fulfilled. Out of the $\mathrm{m} / z$ alignment methods, Kazmi et al. [39] 


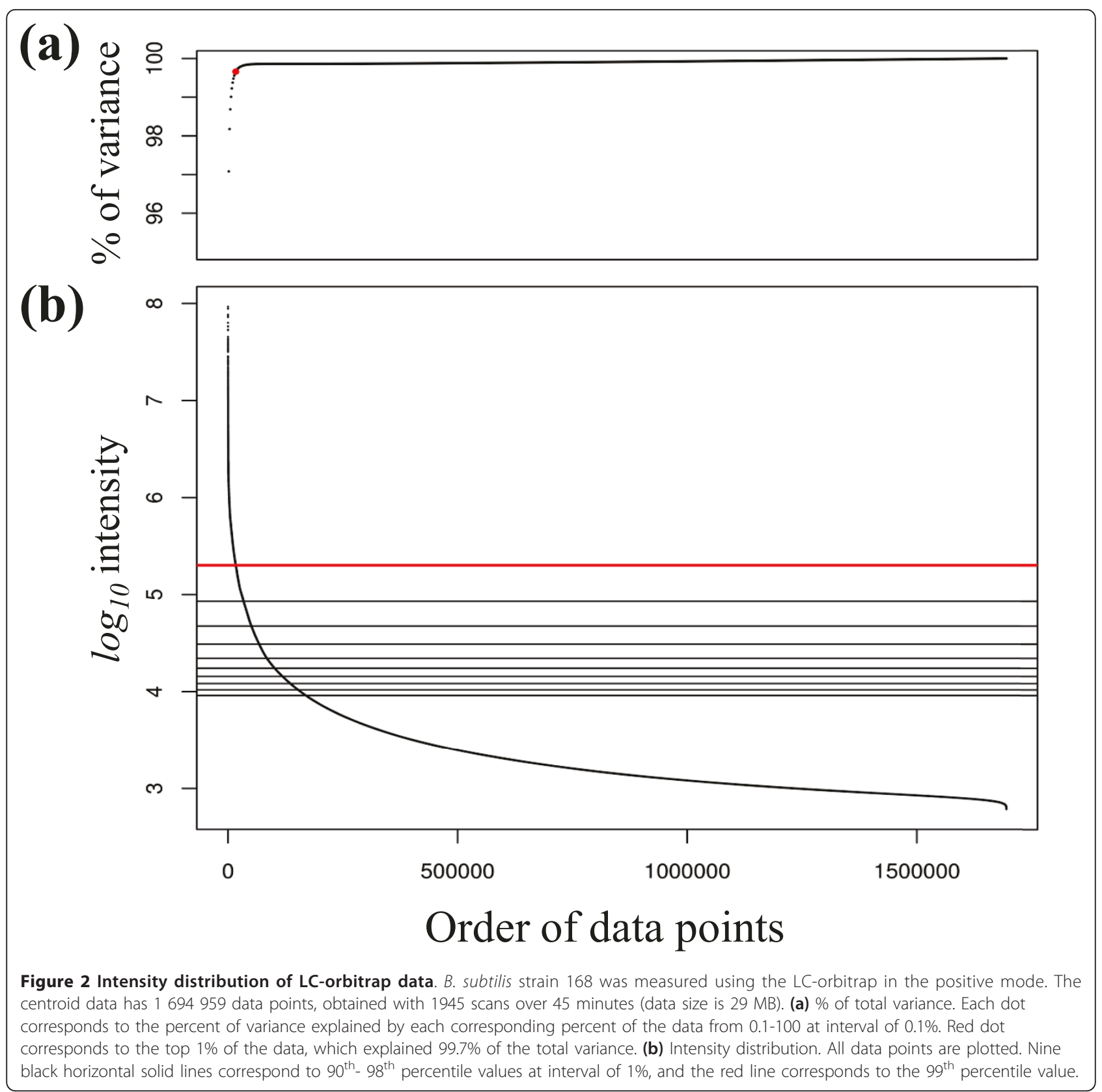

developed the method to create bins and then combine consecutive bins together according to the constrains, similar to complete linkage hierarchical clustering. While they must consider the origins of $\mathrm{m} / \mathrm{z}$ values, our method is to collect all data points with relatively higher intensities and then deal with collected data as one spectrum. Consequently, the grouping of $\mathrm{m} / z$ values is feasible in one step.

Median $\mathrm{m} / \mathrm{z}$ values of individual $\mathrm{m} / \mathrm{z}$ groups are defined as the peak values among all samples. Figure 4 shows the relationship between closeness and the number of $m / z$ groups by simultaneously using two data sets.
In case of closeness $5 \mathrm{ppm}$ (default setting) for the top 1 and $5 \%$ of data points, 624 (black dots in Figure 4) and 2821 (red dots) $m / z$ groups were obtained, respectively. According to Werf et al. [40], the in silico metabolome of B. subtilis is covered by 537 compounds. Of those, 282 compounds are commercially available. Other compounds can not be identified by the method of measuring authentic compounds. Additionally, Pluskal et al. [41] and Iijima et al. [37], for example, identified 123 metabolites from approximately 1900 peaks in yeast and at most 29 metabolites identified by comparison with authentic compounds (they called grade A) from $\sim 4700$ peaks in 


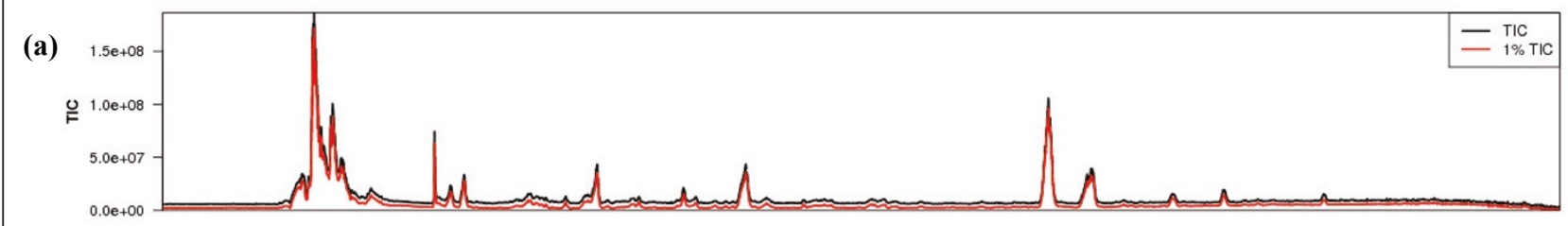

(b)

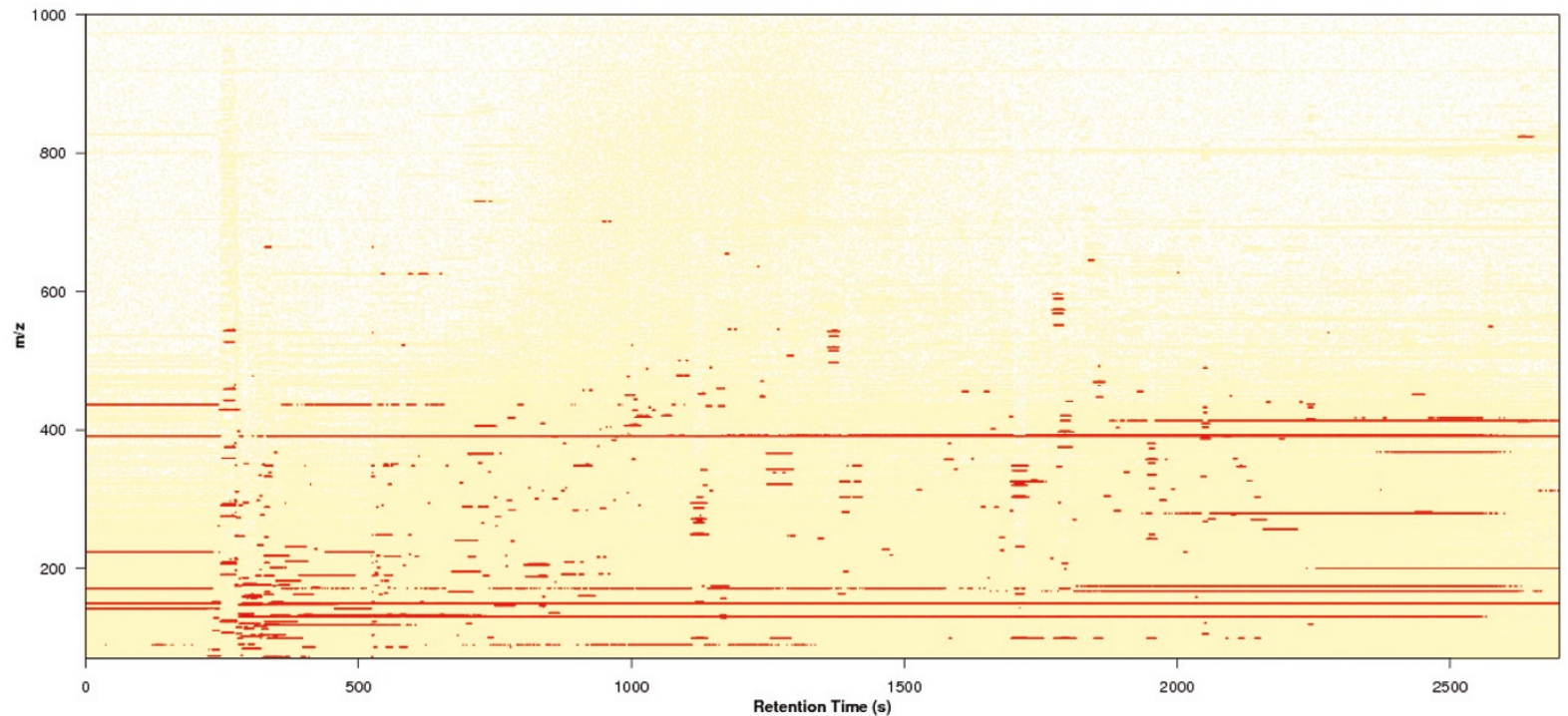

Figure 3 All data vs Top $1 \%$ of data. (a) The comparison of the total ion chromatograms of all and top 1\% of data. The abscissa and ordinate axes correspond to the retention times and ion intensity, respectively. The total ion chromatograms of all data and top 1\% of data were plotted as black and red solid lines, respectively. (b) Two dimensional map ( $\mathrm{m} / \mathrm{z}$ vs retention times). Top $1 \%$ of data are plotted as red points.

tomato, respectively. Those studies indicate that most of obtained peaks from LC-MS data would remain unknown even after peak detection. We concluded that $624 \mathrm{~m} / z$ groups could be sufficient to express the cell state as starting point for LC-orbitrap analysis.

For identification of the ions by $\mathrm{MS}^{2}$ data, we made an in-house database for $B$. subtilis compounds by using KEGG database. All reactions associated with $B$. subtilis were extracted and 890 compounds were set to be as the database (Additional file 1). After database search ([M $+\mathrm{H}]^{+}$) within $\pm 5 \mathrm{ppm}$ for $\mathrm{MS}^{2}$ precursor $m / z s$ in two $B$. subtilis data, 20 available authentic compounds (Additional file 2) were measured under the same conditions for B. subtilis strains. Out of limited $\mathrm{MS}^{2}$ spectra in $B$. subtilis samples, 14 compounds were manually identified by measuring the authentic compounds. Next, we performed a comparison study for $m / z$ accuracy between AMDORAP, MZmine 2 and XCMS. The steps including Chromatogram builder $(m / z$ tolerance $=0.01)$, RANSAC aligner and Peak finder were performed by MZmine 2. In XCMS parameters for UPLC-orbitrap data, Dunn et al. [6] showed that two parameters, snthresh and bw, significantly affected the processed data, e.g., the number of peaks detected and the peak area reproducibility. For XCMS, the parameters were set to be "centWave", $b w=$
60 , snthresh $=2, p p m=3$ and mzwid $=0.02$ with all other default settings. Table 1 summarizes the comparisons of observed $\mathrm{m} / z$ values associated with 14 identified compounds. Seven $m / z$ values obtained by AMDORAP were closest to the theoretical masses. While all errors of observed $m / z$ values in AMDORAP were distributed within $\pm 3 \mathrm{ppm}$, some errors in MZmine 2 and XCMS were over $\pm 100 \mathrm{ppm}$, e.g., tryptophan, uridine and glutamine, suggesting that our procedure can detect more accurate $m / z$ values than others. In the case of other parameter settings for XCMS, a few compounds were not detected (data not shown). In compound searches using mass differences alone, $m / z$ values with errors over \pm 100 ppm could be no longer correctly annotated by leveraging the high-resolution power of the orbitrap. This comparison shows that our method has the best performance in detecting accurate $m / z$ values and can allow us to identify correct candidate compounds by mass differences alone. According to Goerlach et al. [25], 30 and 14 different types of molecular ion adducts exist in the positive and negative modes, respectively. Furthermore, structural isomers and stereoisomers have the same mass. Therefore, it should be noted that putative identification of metabolites based on the accurate $\mathrm{m} / \mathrm{z}$ values is carefully performed to avoid the misleading results. 


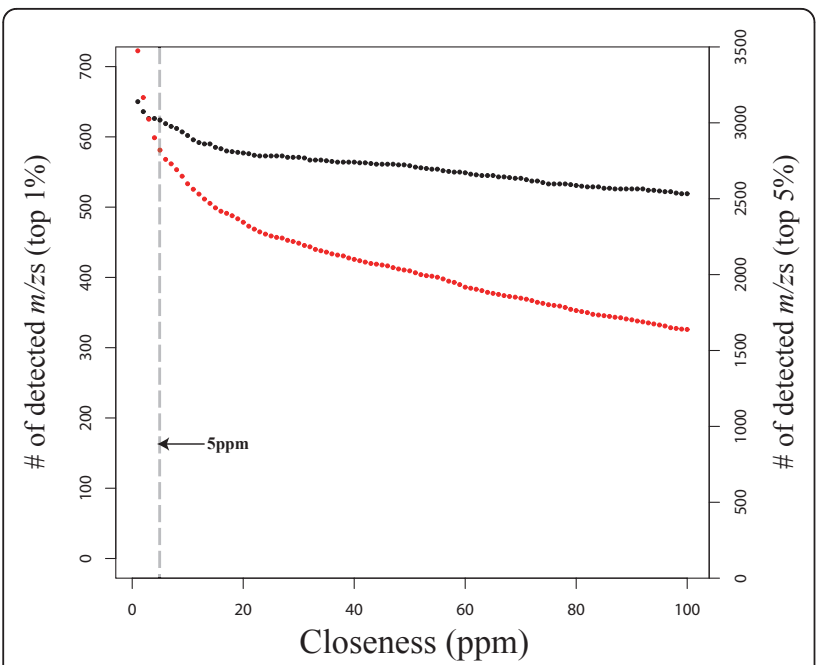

Figure 4 Numbers of detected $\mathrm{m} / \mathrm{z}$ values by the parameter of closeness. Black (ordinate axis on the left) and red (ordinate axis on the right) dots correspond to the numbers of detected $\mathrm{m} / \mathrm{z}$ values using 1 and $5 \%$ of the data, respectively.

\section{Extraction of ion chromatograms for the $\mathrm{m} / \mathrm{z}$ list}

The final step is to extract ion chromatograms for the $\mathrm{m} / \mathrm{z}$ list within $\pm 5 \mathrm{ppm}$ (default setting). AMDORAP provides two types of representative values for detected $\mathrm{m} / \mathrm{z}$ values. One is the sum of total ion chromatogram and another is the sum of selected peak area by a signal-to-noise ratio cutoff for Gaussian filtered chromatogram [42]. Of 624 $\mathrm{m} / \mathrm{z}$ values, 603 reliable chromatograms were extracted by manually checking. We judged the chromatograms with noisy baseline or stretched across the experimental time, i.e., $45 \mathrm{~min}$, as unreliable chromatograms in this study.
Additional file 3 shows 21 extracted ion chromatograms judged to be unreliable chromatograms. The numbers of chromatograms with only one peak through 45 minutes, were 471 (79\%) and $453(75 \%)$ in B. subtilis strains 168 and MGB874, respectively; the numbers of chromatograms with two peaks were $86(14 \%)$ and $113(18 \%)$. As showing in Figure 5, two peaks were seen in a chromatogram of phenylalanine; this phenomenon was confirmed under our experimental conditions by measuring the authentic phenylalanine, indicating that some of the chromatograms with two peaks originate from unique compounds. Those results suggest that to separate the peaks by the retention time could mislead the identification of the ions and clues about the chemical structures corresponding to those peaks could be obtained without separating chromatograms by the retention time. Hence, almost all chromatograms based on AMDORAP could be identified as unique compounds even without separation of identical $\mathrm{m} / \mathrm{z}$ peaks by the retention time. Taken together, the reliable $\mathrm{m} / z$ grouping process is sufficient for comparison of metabolic fingerprinting based on high-resolution LC-MSs.

\section{Conclusions}

In metabolic profiling by the high-resolution mass technologies, it is important to convert raw data to reliable $\mathrm{m} / \mathrm{z}$ values in order to quickly get the information of correct candidate metabolites in biological samples. With respect to obtained $\mathrm{m} / \mathrm{z}$ accuracy, comparison study was performed using only 14 identified compounds. Clearly, the $m / z$ errors by AMDORAP are smallest, although the number of compared compounds might be not enough. In the range of tested parameters,

Table 1 Comparison of detected $\mathrm{m} / \mathrm{z}$ values for fourteen compounds by AMDORAP, MZmine 2 and XCMS

\begin{tabular}{|c|c|c|c|c|c|c|c|c|}
\hline & & & AMDORAP & & MZmine 2 & & XCMS & \\
\hline metabolite & formula & $\begin{array}{l}\text { theoretical } \\
{[\mathrm{M}+\mathrm{H}]^{+}}\end{array}$ & $\begin{array}{l}\text { observed } \\
\mathrm{m} / \mathrm{z}\end{array}$ & $\begin{array}{l}\text { error } \\
\text { ppm }\end{array}$ & $\begin{array}{l}\text { observed } \\
\mathrm{m} / \mathrm{z}\end{array}$ & $\begin{array}{l}\text { error } \\
\text { ppm }\end{array}$ & $\begin{array}{l}\text { Observed } \\
\mathrm{m} / \mathrm{z}\end{array}$ & $\begin{array}{l}\text { error } \\
\text { ppm }\end{array}$ \\
\hline serine & $\mathrm{C}_{3} \mathrm{H}_{7} \mathrm{NO}_{3}$ & 106.04987 & 106.04960 & -2.55 & 106.04955 & -3.02 & 106.04958 & -2.68 \\
\hline valine & $\mathrm{C}_{5} \mathrm{H}_{11} \mathrm{NO}_{2}$ & 118.08626 & 118.08604 & -1.78 & 118.08602 & -2.01 & 118.08422 & -17.26 \\
\hline glutamine & $\mathrm{C}_{5} \mathrm{H}_{10} \mathrm{~N}_{2} \mathrm{O}_{3}$ & 147.07642 & 147.07666 & 1.64 & 147.07642 & 0.03 & 147.14403 & 459.68 \\
\hline lysine & $\mathrm{C}_{6} \mathrm{H}_{14} \mathrm{~N}_{2} \mathrm{O}_{2}$ & 147.11280 & 147.11277 & -0.23 & 147.11067 & -14.49 & 147.10871 & -27.82 \\
\hline glutamic acid & $\mathrm{C}_{5} \mathrm{H}_{9} \mathrm{NO}_{4}$ & 148.06043 & 148.06055 & 0.76 & 148.06042 & -0.09 & 148.05154 & -60.10 \\
\hline methionine & $\mathrm{C}_{5} \mathrm{H}_{11} \mathrm{NO}_{2} \mathrm{~S}$ & 150.05833 & 150.05817 & -1.06 & 150.05818 & -0.96 & 150.04993 & -55.93 \\
\hline D-alanyl-D-alanine & $\mathrm{C}_{6} \mathrm{H}_{12} \mathrm{~N}_{2} \mathrm{O}_{3}$ & 161.09207 & 161.09181 & -1.59 & 161.09197 & -0.60 & 161.08698 & -31.61 \\
\hline phenylalanine & $\mathrm{C}_{9} \mathrm{H}_{11} \mathrm{NO}_{2}$ & 166.08626 & 166.08617 & -0.53 & 166.08618 & -0.44 & 166.08285 & -20.50 \\
\hline citrulline & $\mathrm{C}_{6} \mathrm{H}_{13} \mathrm{~N}_{3} \mathrm{O}_{3}$ & 176.10297 & 176.10280 & -0.96 & 176.10281 & -0.87 & 176.11662 & 77.51 \\
\hline tyrosine & $\mathrm{C}_{9} \mathrm{H}_{11} \mathrm{NO}_{3}$ & 182.08117 & 182.08113 & -0.21 & 182.08110 & -0.41 & 182.08119 & 0.09 \\
\hline tryptophan & $\mathrm{C}_{11} \mathrm{H}_{12} \mathrm{~N}_{2} \mathrm{O}_{2}$ & 205.09715 & 205.09732 & 0.81 & 205.06284 & -167.29 & 205.09010 & -34.38 \\
\hline pantothenate & $\mathrm{C}_{9} \mathrm{H}_{17} \mathrm{NO}_{5}$ & 220.11795 & 220.11798 & 0.14 & 220.11414 & -17.29 & 220.12327 & 24.18 \\
\hline uridine & $\mathrm{C}_{9} \mathrm{H}_{12} \mathrm{~N}_{2} \mathrm{O}_{6}$ & 245.07681 & 245.07692 & 0.44 & 245.10884 & 130.69 & 245.08308 & 25.58 \\
\hline methylthioadenosine & $\mathrm{C}_{11} \mathrm{H}_{15} \mathrm{~N}_{5} \mathrm{O}_{3} \mathrm{~S}$ & 298.09684 & 298.09708 & 0.80 & 298.08231 & -48.72 & 298.09719 & 1.18 \\
\hline
\end{tabular}

Comparison of detected $m / z$ values between AMDORAP, MZmine 2 and XCMS. Seven $m / z$ values obtained by AMDORAP, i.e., serine, valine, lysine, tryptophan, pantothenate, uridine and methylthioadenosine, were closest to the theoretical masses. 


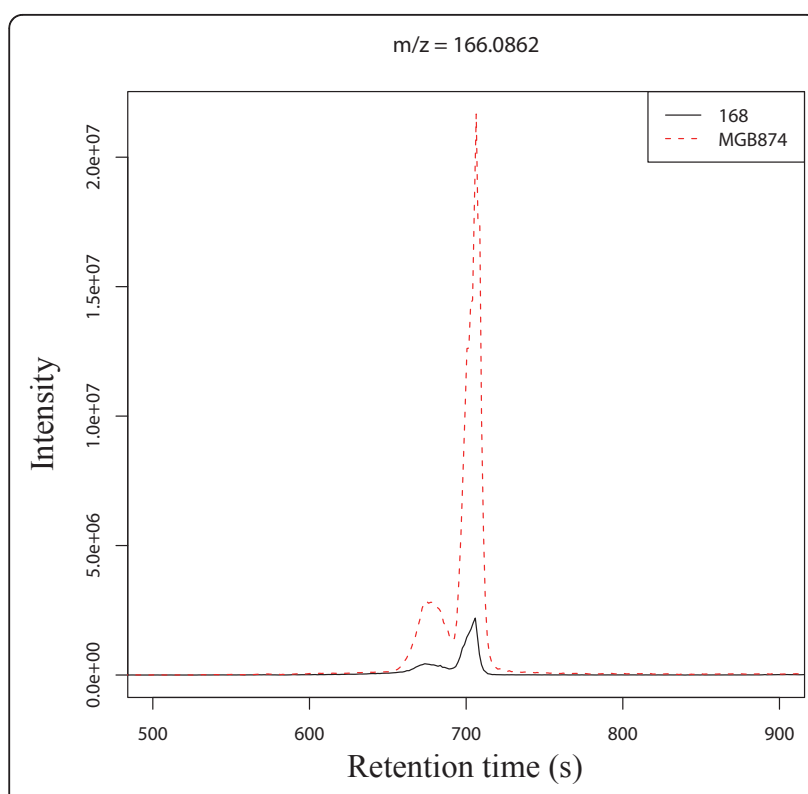

Figure 5 A chromatogram trace of phenylalanine. Chromatogram trace of $\mathrm{m} / \mathrm{z}$ slice $166.08534-166.08700$ is presented. Black solid and red dashed lines correspond to B. subtilis strains 168 and MGB874, respectively. Two peak areas were also observed for an authentic compound under our conditions.

we couldn't get better results for 14 compounds by MZmine 2 and XCMS. This suggests that parameter optimization of those tools is time consuming process and difficult to find out best settings for both dimensions, i.e., $m / z$ and retention time. Furthermore, it would suggest that both mass and retention time alignment processes introduce the larger errors for obtained $m / z$ values, while AMDORAP uses only the ions with relatively higher intensities for estimating the $\mathrm{m} / \mathrm{z}$ values. In addition, a signal-to-noise ratio cutoff by Gaussian filtering could allow us to achieve a reliable comparison of the ion abundances between samples, even when there are peaks with noisy baseline. Thus, AMDORAP can detect more accurate $m / z$ values from raw data and provide the platform for metabolic fingerprinting. Information of $\mathrm{MS}^{n}$, retention time and behaviors of the authentic compounds has the essential roles to finally verify the ions as particular metabolites. However, the extraction of interesting accurate $\mathrm{m} / \mathrm{z}$ values by AMDORAP should be indispensable as a starting point for comparative LC-orbitrap analysis, because of the limitations of available authentic compounds and simultaneously obtained $\mathrm{MS}^{2}$ spectra with a full MS scan per sample.

\section{Availability and requirements \\ Project name: AMDORAP \\ Project home page: http://amdorap.sourceforge.net/ \\ Operating systems: Platform independent}

\author{
Programming language: $\mathrm{R}$ \\ License: GPL v2
}

Any restrictions to use by non-academics: No

\section{Additional material}

Additional file 1: A list of $\mathbf{8 9 0}$ compounds. This list contains 890 compounds associated with all reactions in $B$. subtilis of KEGG.

Additional file 2: A list of $\mathbf{2 0}$ authentic compounds. These compounds were measured by LC-orbitrap. Obtained information (MS ${ }^{2}$ and retention time) were used to identify the compounds.

Additional file 3: 21 unreliable chromatograms. 21 extracted ion chromatograms judged to be unreliable chromatograms are shown. The abscissa and ordinate axes correspond to the retention times and ion intensity, respectively.

\section{Acknowledgements}

This work has been partly supported by the Japan Science and Technology Agency, CREST (Elucidation of Amino Acid Metabolism in Plants Based on Integrated Omics Analyses). B. subtilis strain MGB874 was created by the New Energy and Industrial Technology Development Organization, NEDO (Development of a Technology for the Creation of a Host Cell). We thank the Plant Global Education Program of Nara Institute of Science and Technology for the use of the LC-orbitrap. We also would like to acknowledge Dr. Kazuki Saito of RIKEN Plant Science Center for offering the authentic compounds.

\section{Author details}

${ }^{1}$ Graduate School of Information Science, Nara Institute of Science and Technology, 8916-5 Takayama, Ikoma, Nara 630-0192, Japan. ²Biological Science Laboratories, Kao Corporation, 2606 Akabane, Ichikai, Haga, Tochigi 321-3497, Japan.

\section{Authors' contributions}

HT performed experimental parts, implemented the methods, analyzed data sets and wrote the manuscript. TM performed extraction of metabolites. NO and SK supervised this project. All authors have read and approved the final manuscript.

\section{Competing interests}

The authors declare that they have no competing interests.

Received: 15 February 2011 Accepted: 24 June 2011

Published: 24 June 2011

\section{References}

1. Hall RD: Plant metabolomics: from holistic hope, to hype, to hot topic. New Phytol 2006, 169(3):453-468.

2. Fiehn O, Kopka J, Dormann P, Altmann T, Trethewey RN, Willmitzer L: Metabolite profiling for plant functional genomics. Nat Biotechnol 2000, 18(11):1157-1161.

3. Hirai MY, Yano M, Goodenowe DB, Kanaya S, Kimura T, Awazuhara M, Arita M, Fujiwara T, Saito K: Integration of transcriptomics and metabolomics for understanding of global responses to nutritional stresses in Arabidopsis thaliana. Proc Natl Acad Sci USA 2004, 101(27):10205-10210.

4. Hirai MY, Klein M, Fujikawa Y, Yano M, Goodenowe DB, Yamazaki Y, Kanaya S, Nakamura Y, Kitayama M, Suzuki H, Sakurai N, Shibata D,

Tokuhisa J, Reichelt M, Gershenzon J, Papenbrock J, Saito K: Elucidation of gene-to-gene and metabolite-to-gene networks in arabidopsis by integration of metabolomics and transcriptomics. J Biol Chem 2005, 280(27):25590-25595.

5. Takahashi H, Morioka R, Ito R, Oshima T, Altaf-Ul-Amin M, Ogasawara N, Kanaya S: Dynamics of Time-Lagged Gene-to-Metabolite Networks of Escherichia coli Elucidated by Integrative Omics Approach. OMICS 2011, 15(1-2):15-23. 
6. Dunn WB, Broadhurst D, Brown M, Baker PN, Redman CW, Kenny LC, Kell DB: Metabolic profiling of serum using Ultra Performance Liquid Chromatography and the LTQ-Orbitrap mass spectrometry system. J Chromatogr B Analyt Technol Biomed Life Sci 2008, 871(2):288-298.

7. Brown M, Wedge DC, Goodacre R, Kell DB, Baker PN, Kenny LC, Mamas MA, Neyses L, Dunn WB: Automated work flows for accurate mass-based putative metabolite identification in LC/MS-derived metabolomic datasets. Bioinformatics 2011, 27(8):1108-1112.

8. Dunn WB, Broadhurst DI, Atherton HJ, Goodacre R, Griffin JL: Systems level studies of mammalian metabolomes: the roles of mass spectrometry and nuclear magnetic resonance spectroscopy. Chem Soc Rev 2011, 40:387-426.

9. Verhoeven HA, de Vos CHR, Bino RJ, Hall RD: Plant metabolomics strategies based upon Quadruple Time of Flight Mass Spectrometry (QTOF-MS). In Plant Metabolomics. Biotechnology in agriculture and forestry. Volume 57. Springer; 2006:33-48.

10. Scigelova M, Makarov A: Orbitrap mass analyzer-overview and applications in proteomics. Proteomics 2006, 6(Suppl 2):16-21.

11. Han X, Aslanian A, Yates JRr: Mass spectrometry for proteomics. Curr Opin Chem Biol 2008, 12(5):483-490.

12. Perry RH, Cooks RG, Noll RJ: Orbitrap mass spectrometry: instrumentation, ion motion and applications. Mass Spectrom Rev 2008, 27(6):661-699.

13. Zhang NR, Yu S, Tiller P, Yeh S, Mahan E, Emary WB: Quantitation of small molecules using high-resolution accurate mass spectrometers - a different approach for analysis of biological samples. Rapid Commun Mass Spectrom 2009, 23(7):1085-1094.

14. Marshall AG, Hendrickson CL: High-resolution mass spectrometers. Annu Rev Anal Chem (Palo Alto Calif) 2008, 1:579-599.

15. Breitling R, Pitt AR, Barrett MP: Precision mapping of the metabolome. Trends Biotechnol 2006, 24(12):543-548.

16. Lu W, Clasquin MF, Melamud E, Amador-Noguez D, Caudy AA, Rabinowitz JD: Metabolomic analysis via reversed-phase ion-pairing liquid chromatography coupled to a stand alone orbitrap mass spectrometer. Anal Chem 2010, 82(8):3212-3221.

17. Degtyarenko K, de Matos P, Ennis M, Hastings J, Zbinden M, McNaught A, Alcantara R, Darsow M, Guedj M, Ashburner M: ChEBl: a database and ontology for chemical entities of biological interest. Nucleic Acids Res 2008, , 36 Database: D344-D350.

18. Wishart DS, Tzur D, Knox C, Eisner R, Guo AC, Young N, Cheng D, Jewell K Arndt D, Sawhney S, Fung C, Nikolai L, Lewis M, Coutouly MA, Forsythe I, Tang P, Shrivastava S, Jeroncic K, Stothard P, Amegbey G, Block D, Hau DD, Wagner J, Miniaci J, Clements M, Gebremedhin M, Guo N, Zhang Y, Duggan GE, Macinnis GD, Weljie AM, Dowlatabadi R, Bamforth F, Clive D, Greiner R, Li L, Marrie T, Sykes BD, Vogel HJ, Querengesser L: HMDB: the Human Metabolome Database. Nucleic Acids Res 2007, , 35 Database: D521-D526.

19. KEGG. [http://www.genome.jp/KEGG/]

20. Shinbo Y, Nakamura Y, Altaf-UI-Amin M, Asahi H, Kurokawa K, Arita M, Saito K, Ohta D, Shibata D, Kanaya S: KNApSAcK: A Comprehensive Species-Metabolite Relationship Database. In Plant Metabolomics. Biotechnology in agriculture and forestry. Volume 57. Springer; 2006:165-181.

21. PubChem. [http://pubchem.ncbi.nlm.nih.gov/].

22. Oikawa A, Nakamura Y, Ogura T, Kimura A, Suzuki H, Sakurai N, Shinbo Y, Shibata D, Kanaya S, Ohta D: Clarification of pathway-specific inhibition by Fourier transform ion cyclotron resonance/mass spectrometry-based metabolic phenotyping studies. Plant Physiol 2006, 142(2):398-413.

23. Takahashi H, Kai K, Shinbo Y, Tanaka K, Ohta D, Oshima T, Altaf-Ul-Amin M, Kurokawa K, Ogasawara N, Kanaya S: Metabolomics approach for determining growth-specific metabolites based on Fourier transform ion cyclotron resonance mass spectrometry. Anal Bioanal Chem 2008, 391(8):2769-2782.

24. Huanga N, Siegela MM, Kruppab HG, Laukienb HF: Automation of a Fourier transform ion cyclotron resonance mass spectrometer for acquisition, analysis, and e-mailing of high-resolution exact-mass electrospray ionization mass spectral data. J Am Soc Mass Spectr 1999, 10(11):1166-1173.

25. Goerlach E, Richmond R: Discovery of Quasi-Molecular lons in Electrospray Spectra by Automated Searching for Simultaneous Adduct Mass Differences. Anal Chem 1999, 71(24):5557-5562.
26. Allen J, Davey HM, Broadhurst D, Heald JK, Rowland JJ, Oliver SG, Kell DB: High-throughput classification of yeast mutants for functional genomics using metabolic footprinting. Nat Biotechnol 2003, 21(6):692-696.

27. Fiehn O: Metabolomics-the link between genotypes and phenotypes. Plant Mol Biol 2002, 48(1-2):155-171.

28. Katajamaa $M$, Oresic $M$ : Data processing for mass spectrometry-based metabolomics. J Chromatogr A 2007, 1158(1-2):318-328.

29. Katajamaa M, Oresic M: Processing methods for differential analysis of LC/MS profile data. BMC Bioinformatics 2005, 6:179.

30. Pluskal T, Castillo S, Villar-Briones A, Oresic M: MZmine 2: modular framework for processing, visualizing, and analyzing mass spectrometrybased molecular profile data. BMC Bioinformatics 2010, 11:395.

31. Smith CA, Want EJ, O'Maille G, Abagyan R, Siuzdak G: XCMS: processing mass spectrometry data for metabolite profiling using nonlinear peak alignment, matching, and identification. Anal Chem 2006, 78(3):779-787.

32. Tautenhahn R, Bottcher C, Neumann S: Highly sensitive feature detection for high resolution LC/MS. BMC Bioinformatics 2008, 9:504.

33. R Development Core Team: R: A Language and Environment for Statistical Computing R Foundation for Statistical Computing, Vienna, Austria; 2008 [http://www.R-project.org], [ISBN 3-900051-07-0].

34. Morimoto T, Kadoya R, Endo K, Tohata M, Sawada K, Liu S, Ozawa T, Kodama T, Kakeshita H, Kageyama Y, Manabe K, Kanaya S, Ara K, Ozaki K, Ogasawara N: Enhanced recombinant protein productivity by genome reduction in Bacillus subtilis. DNA Res 2008, 15(2):73-81.

35. Anagnostopoulos C, Spizizen J: Requirements for Transformation In Bacillus Subtilis. J Bacteriol 1961, 81(5):741-746.

36. Harwood CR, Archibald AR: Growth, maintenance and general techniques. In Molecular Biological Methods for Bacillus. Edited by: Harwood CR, Cutting SM. John Wiley 1990:549.

37. lijima Y, Nakamura Y, Ogata Y, Tanaka K, Sakurai N, Suda K, Suzuki T, Suzuki H, Okazaki K, Kitayama M, Kanaya S, Aoki K, Shibata D: Metabolite annotations based on the integration of mass spectral information. Plant J 2008, 54(5):949-962.

38. Keller A, Eng J, Zhang N, Li XJ, Aebersold R: A uniform proteomics MS/MS analysis platform utilizing open XML file formats. Mol Syst Biol 2005, 1, 2005.0017.

39. Kazmi AS, Ghosh S, Shin DG, Hill WD, Grant FD: Alignment of high resolution mass spectra: development of a heuristic approach for metabolomics. Metabolomics 2006, 2(2):75-83.

40. van der Werf MJ, Overkamp KM, Muilwijk B, Coulier L, Hankemeier T: Microbial metabolomics: toward a platform with full metabolome coverage. Anal Biochem 2007, 370:17-25.

41. Pluskal T, Nakamura T, Villar-Briones A, Yanagida M: Metabolic profiling of the fission yeast $\mathrm{S}$. pombe: quantification of compounds under different temperatures and genetic perturbation. Mol Biosyst 2010, 6:182-198.

42. Danielsson R, Bylund D, Markides KE: Matched filtering with background suppression for improved quality of base peak chromatograms and mass spectra in liquid chromatography-mass spectrometry. Analytica Chimica Acta 2002, 454(2):167-184.

doi:10.1186/1471-2105-12-259

Cite this article as: Takahashi et al: AMDORAP: Non-targeted metabolic profiling based on high-resolution LC-MS. BMC Bioinformatics 2011 12:259.

\section{Submit your next manuscript to BioMed Central and take full advantage of:}

- Convenient online submission

- Thorough peer review

- No space constraints or color figure charges

- Immediate publication on acceptance

- Inclusion in PubMed, CAS, Scopus and Google Scholar

- Research which is freely available for redistribution

Submit your manuscript at www.biomedcentral.com/submit
C Biomed Central 